https://doi.org/10.15407/ujpe65.12.1094

B. HEINZ, ${ }^{1,2}$ Q. WANG ${ }^{1,3}$ R. VERBA, ${ }^{4}$ V.I. VASYUCHKA, ${ }^{1}$ M. KEWENIG ${ }^{1}$

P. PIRRO,$^{1}$ M. SCHNEIDER ${ }^{1}$ T. MEYER ${ }^{1,5}$ B. LÄGEL,${ }^{6}$ C. DUBS ${ }^{7}$ T. BRÄCHER ${ }^{1}$ O.V. DOBROVOLSKIY, ${ }^{3}$ A.V. CHUMAK ${ }^{1,3}$

${ }^{1}$ Fachbereich Physik and Landesforschungszentrum OPTIMAS, Technische Universität Kaiserslautern (Erwin-Schrödinger-Straße 56, 67663 Kaiserslautern, Germany)

2 Graduate School Materials Science in Mainz (Staudingerwerg 9, 55128 Mainz, Germany)

3 Nanomagnetism and Magnonics, Faculty of Physics, University of Vienna (Boltzmanngasse 5, A-1090Wien, Austria; e-mail: andrii.chumak@univie.ac.at)

${ }^{4}$ Institute of Magnetism (36-b, Vernadskogo Blvd., Kyiv 03142, Ukraine)

5 THATec Innovation $\mathrm{GmbH}$ (Augustaanlage 23, 68165 Mannheim, Germany)

${ }^{6}$ Nano Structuring Center, Technische Universität Kaiserslautern (Erwin-Schrödinger-Straße 13, 67663 Kaiserslautern, Germany)

7 INNOVENT e.V., Technologieentwicklung (Prüssingstraße 27B, 07745 Jena, Germany)

\title{
TEMPERATURE DEPENDENCE OF SPIN PINNING AND SPIN-WAVE DISPERSION IN NANOSCOPIC FERROMAGNETIC WAVEGUIDES
}

The field of magnonics attracts significant attention due to the possibility of utilizing information coded into the spin-wave phase or amplitude to perform computation operations on the nanoscale. Recently, spin waves were investigated in Yttrium Iron Garnet (YIG) waveguides with widths down to $50 \mathrm{~nm}$ and aspect ratios of thickness to width approaching unity. A critical width was found, below which the exchange interaction suppresses the dipolar pinning phenomenon, and the system becomes unpinned. Here, we continue these investigations and analyze the pinning phenomenon and spin-wave dispersion as functions of temperature, thickness, and material parameters. Higher order modes, the influence of a finite wavevector along the waveguide, and the impact of the pinning phenomenon on the spin-wave lifetime are discussed, as well as the influence of a trapezoidal cross-section and edge roughness of the waveguide. The presented results are of particular interest for potential applications in magnonic devices and the incipient field of quantum magnonics at cryogenic temperatures.

Keywords: spin waves, yttrium iron garnet, Brillouin light scattering spectroscopy, low temperatures.

\section{Introduction}

The field of magnonics proposes a promising approach for a novel type of computing systems, in which

(C) B. HEINZ, Q. WANG, R. VERBA, V.I. VASYUCHKA, M. KEWENIG, P. PIRRO, M. SCHNEIDER,

T. MEYER, B. LÄGEL, C. DUBS, T. BRÄCHER,

O.V. DOBROVOLSKIY, A.V. CHUMAK, 2020 magnons, the quanta of spin waves, carry the information instead of electrons [1-13]. Since the phase of a spin wave provides an additional degree of freedom, efficient computing concepts can be used resulting in a valuable decrease in the footprint of logic units. Moreover, the scalability of magnonic structures down to the nanometer scale and the possibility to operate with spin waves of nanometer wave-

ISSN 2071-0194. Ukr. J. Phys. 2020. Vol. 65, No. 12 
lengths are additional advantages of the magnonics approach. The further miniaturization will, consequently, result in an increase in the frequency of spin waves used in the devices from the currently employed $\mathrm{GHz}$ range up to the $\mathrm{THz}$ range. In classical magnonics, spin-wave modes in thin films or rather planar waveguides with thickness-to-width aspect ratios $a_{\mathrm{r}}=h / w \ll 1$ have been utilized. In the case of a waveguide, edge magnetostatic charges arise, which can be accounted for by the introduction of boundary conditions [14]. Therefore, thin waveguides demonstrate the effect of "dipolar pinning" at the lateral edges, and, for its theoretical description, the thin strip approximation was developed, in which only the pinning of the much-larger-in-amplitude dynamic inplane magnetization component is taken into account [15-20].

The recent progress in the fabrication technology leads to the development of nanoscopic magnetic devices in which the width $w$ and the thickness $h$ become comparable [21-29]. The description of such waveguides is beyond the thin strip model of effective pinning, because the scale of nonuniformity of the dynamic dipolar fields, which is described as "effective dipolar boundary conditions", becomes comparable to the waveguide width. Additionally, both, in-plane and out-of-plane dynamic magnetization components, become involved in the effective dipolar pinning, as they become of comparable amplitude. Thus, a more general model should be developed and verified experimentally. In addition, such nanoscopic feature sizes imply that the spinwave modes bear a strong exchange character, since the widths of the structures are now comparable to the exchange length [30]. A proper description of the spin-wave eigenmodes in nanoscopic strips which considers the influence of the exchange interaction, as well as the shape of the structure, was recently performed in [31] and is fundamental for the field of magnonics.

Very recently, the fields of quantum magnonics and magnonics at cryogenic temperatures were established. Among the highlights, one should mention the first realization of coherent coupling between a ferromagnetic magnon and a superconducting qubit [32], the first observation of the interaction between magnons and Abrikosov fluxes in superconductorferromagnet hybrid structures [33], the investigation of the interplay of the magnetization dynam- ics with a microwave waveguide at cryogenic temperatures [34] and many more interesting phenomena [35-37]. Thus, the understanding of the influence of the temperature on spin pinning conditions and on the spin-wave dispersion in nanostructures is of high demand.

Here, we continue the investigation carried out in Phys. Rev. Lett. 122, 247202 (2019). The evolution of the frequencies and profiles of the spin-wave modes in Yttrium Iron Garnet (YIG) waveguides with a thickness of $39 \mathrm{~nm}$ and widths down to $50 \mathrm{~nm}$ are discussed in detail. The phenomenon of unpinning and the underlying theory, as well as the experimental proof, are outlined. A thorough discussion of the effective width and the critical width, at which the system becomes unpinned, in dependence on the thickness and the material of choice is presented. Moreover, the temperature dependence is analyzed theoretically. Higher order modes up to $n=2$, the influence of a finite wavevector along the waveguide and the impact of the pinning phenomenon on the spin wave lifetime are discussed. To account for the imperfections of a real system, the influence of a trapezoidal cross-section and edge roughness on the effective width and the critical width are investigated.

\section{Methodology}

\subsection{Sample fabrication}

A $39 \mathrm{~nm}$ thick Yttrium Iron Garnet (YIG) film has been grown on a 1 inch (111) $500 \mu \mathrm{m}$ thick Gadolinium Gallium Garnet (GGG) substrate by the liquid phase epitaxy from $\mathrm{PbO}-\mathrm{B}_{2} \mathrm{O}_{3}$ based hightemperature solutions at $860{ }^{\circ} \mathrm{C}$ using the isothermal dipping method (see, e.g., Ref. [38]). A pure $\mathrm{Y}_{3} \mathrm{Fe}_{5} \mathrm{O}_{12}$ film with a smooth surface was obtained by rotating the substrate horizontally with a rotation rate of $100 \mathrm{rpm}$. The saturation magnetization of the YIG film is $1.37 \times 10^{5} \mathrm{~A} / \mathrm{m}$, its Gilbert damping is $\alpha=6.41 \times 10^{-4}$ and the inhomogeneous linewidth is $\mu_{0} \Delta H_{0}=0.001 \mathrm{mT}$, as it was extracted by ferromagnetic resonance spectroscopy [39].

The nanostructures were fabricated by utilizing a hard mask ion milling procedure. The key steps in the fabrication process are shown in Fig. 1, a. First, a double layer of polymethyl methacrylate (PMMA) was spin-coated on the YIG film and a chromium/titanium hard mask was fabricated using electron beam 

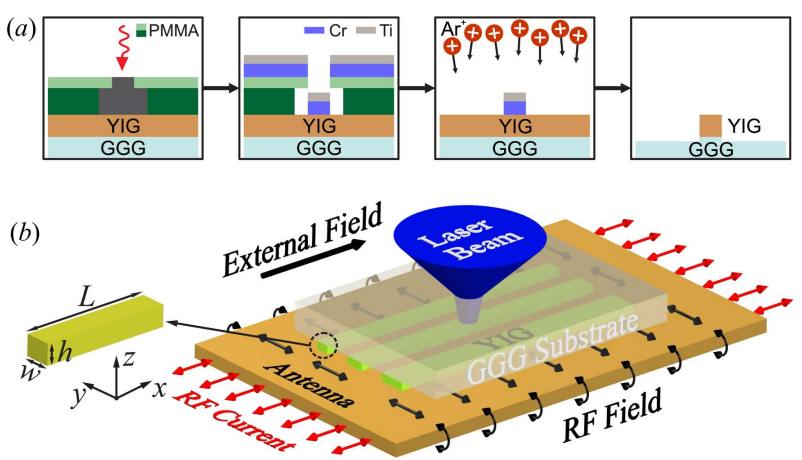

(c)

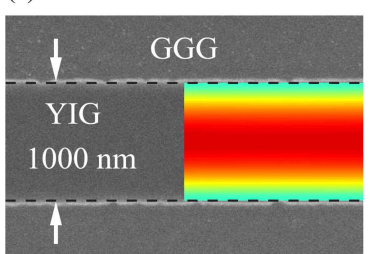

(d)

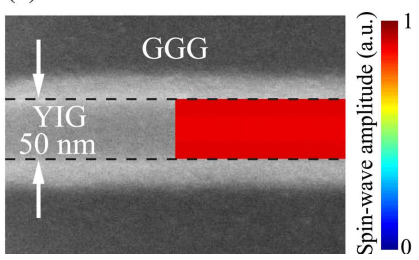

Fig. 1. Schematically depicted main steps in the nanostructuring process $(a)$. Sketch of the sample and the experimental configuration: a set of YIG waveguides is placed on a microstrip line to excite the quasi-FMR in the waveguides. BLS spectroscopy is used to measure the local spin-wave dynamics (b). SEM micrograph of a $1 \mu \mathrm{m}$ and a $50-\mathrm{nm}$ wide YIG waveguide of $39-\mathrm{nm}$ thickness. The color code shows the simulated amplitudes of the fundamental mode at the quasiferromagnetic resonance, i.e., $k_{x}=0$, in the waveguides. The mode in the $50 \mathrm{~nm}$ waveguide is almost uniform across the width of the waveguide evidencing the unpinning directly $(c)$ and $(d)$ [31]

lithography and electron beam evaporation. This hard mask acts as a protective layer in a successive $\mathrm{Ar}^{+}$ion milling step. In a final step, any residual chromium is removed using an acid that YIG is inert to.

\subsection{Microfocused Brillouin Light Scattering (BLS) spectroscopy measurements}

BLS spectroscopy is a unique technique for measuring the spin-wave intensities in frequency, space, and time domains. It is based on the inelastic scattering of an incident laser beam from a magnetic material. In our measurements, a laser beam of $457 \mathrm{~nm}$ wavelength and a power of $1.8 \mathrm{~mW}$ is focused through the transparent GGG substrate on the center of the respective individual waveguide using a $100 \times$ microscope objective with a large numerical aperture $(\mathrm{NA}=0.85)$. The effective spot-size is $350 \mathrm{~nm}$. The scattered light was collected and guided into a six- pass Fabry-Pérot interferometer to analyze the frequency shift.

\subsection{Numerical simulations}

The micromagnetic simulations of the space- and time-dependent magnetization dynamics were performed by the GPU-accelerated simulation program Mumax3 using a finite-difference discretization [40]. The structure is schematically shown in Fig. $1, b$. The following material parameters were used in the simulations: the saturation magnetization $M_{\mathrm{S}}=$ $=1.37 \times 10^{5} \mathrm{~A} / \mathrm{m}$ and the Gilbert damping $\alpha=$ $=6.41 \times 10^{-4}$ were extracted by ferromagnetic resonance spectroscopy measurements of the plain film before the patterning [41]. The gyromagnetic ratio $\gamma=175.86 \mathrm{rad} /(\mathrm{ns} \cdot \mathrm{T})$ and the exchange constant $A=3.5 \mathrm{pJ} / \mathrm{m}$ for a standard YIG film were assumed. The external field $B=108.9 \mathrm{mT}$ was applied along the waveguide long axis. Three steps were performed to calculate the spin-wave dispersion curve: (i) The external field was applied along the waveguide, and the magnetization was allowed to relax into a stationary state (ground state). (ii) A sinc field pulse $b_{y}=b_{0} \operatorname{sinc}\left(2 \pi f_{c} t\right)$, with the oscillation field $b_{0}=1 \mathrm{mT}$ and cut-off frequency $f_{c}=10 \mathrm{GHz}$, was used to excite spin waves in a wide range. (iii) The spin-wave dispersion relations were obtained by performing the two-dimensional Fast Fourier Transformation of the time- and spacedependent data. Furthermore, the spin-wave width profiles were extracted from the $m_{z}$ component across the width of the waveguides using a single frequency excitation.

\subsection{Quasianalytic theoretical model}

In order to accurately describe the spin-wave characteristics in nanoscopic longitudinally magnetized waveguides, a more general semianalytic theory is provided which goes beyond the thin strip approximation [31]. Here, we assume a uniform spin wave mode profile across the waveguide thickness (no $z$ dependence), which is valid for the fundamental thickness mode in thin waveguides. In a general case, the $z$-dependence of any higher thickness mode can be included in a similar way as shown here. Please note that the theory is not applicable in transversely magnetized waveguides due to their more involved internal field landscape [22]. The lateral spin-wave

ISSN 2071-0194. Ukr. J. Phys. 2020. Vol. 65, No. 12 
mode profile $\mathbf{m}_{k_{x}}(y)$ and frequency can be found as solutions of the linearized Landau-Lifshitz equation $[42,43]$

$-i \omega_{k_{x}} \mathbf{m}_{k_{x}}(y)=\boldsymbol{\mu} \times\left(\hat{\Omega}_{k_{x}} \cdot \mathbf{m}_{k_{x}}(y)\right)$,

with appropriate exchange boundary conditions, which take into account the surface anisotropy at the edges. Here, $\boldsymbol{\mu}$ is the unit vector in the static magnetization direction, and $\hat{\Omega}_{k_{x}}$ is a tensorial Hamilton operator, which is given by

$\hat{\Omega}_{k_{x}} \cdot \mathbf{m}_{k_{x}}(y)=\left(\omega_{\mathrm{H}}+\omega_{\mathrm{M}} \lambda^{2}\left(k_{x}^{2}-\frac{d^{2}}{d y^{2}}\right)\right) \mathbf{m}_{k_{x}}(y)+$

$+\omega_{\mathrm{M}} \int \hat{\mathbf{G}}_{k_{x}}\left(y-y^{\prime}\right) \cdot \mathbf{m}_{k_{x}}\left(y^{\prime}\right) d y^{\prime}$.

Here, $\omega_{\mathrm{H}}=\gamma B, B$ is the static internal magnetic field that is considered to be equal to the external field due to the negligible demagnetization along the $x$ direction, $\omega_{\mathrm{M}}=\gamma \mu_{0} M_{\mathrm{s}}, \gamma$ is the gyromagnetic ratio, and $\hat{\mathbf{G}}_{k_{x}}$ is a Green's function (see next subsection).

A numerical solution of Eq. (1) gives both, the spinwave profile $\mathbf{m}_{k_{x}}$ and frequency $\omega_{k_{x}}$. In the following, we will regard the ouf-of-plane component $m_{z}(y)$ to show the mode profiles representatively. In the past, it was demonstrated that in microscopic waveguides, the fundamental mode is well fitted by the function $m_{z}(y)=A_{0} \cos \left(\pi y / w_{\text {eff }}\right)$ with the amplitude $A_{0}$ and the effective width $w_{\text {eff }}[17,18]$. This mode, as well as the higher modes, are referred to as "partially pinned". Pinning hereby is related to the fact that the amplitude of the mode at the edges of the waveguide is reduced. In that case, the effective width $w_{\text {eff }}$ determines where the amplitude of the modes would vanish outside the waveguide $[9,17,29]$. With this effective width, the spin-wave dispersion relation can also be calculated by the analytic formula [9]:

$\omega_{0}\left(k_{x}\right)=$

$=\sqrt{\left(\omega_{\mathrm{H}}+\omega_{\mathrm{M}}\left(\lambda^{2} K^{2}+F_{k_{x}}^{y y}\right)\right)\left(\omega_{\mathrm{H}}+\omega_{\mathrm{M}}\left(\lambda^{2} K^{2}+F_{k_{x}}^{z z}\right)\right)}$,

where $K=\sqrt{k_{x}^{2}+\kappa^{2}}$ and $\kappa=\pi / w_{\text {eff. }}$ The tensor $\hat{\mathbf{F}}_{k_{x}}=\frac{1}{2 \pi} \int_{-\infty}^{\infty} \frac{\left|\sigma_{k}\right|^{2}}{\tilde{w}} \hat{\mathbf{N}}_{k} d k_{y}$ accounts for the dynamic magnetization, $\sigma_{k}=\int_{-w / 2}^{w / 2} m(y) e^{-i k_{y} y} d y$ is the Fourier-transform of the spin-wave profile across the width of the waveguide, and $\tilde{w}=\int_{-w / 2}^{w / 2} m(y)^{2} d y$ is the normalization of the mode profile $m(y)$.

ISSN 2071-0194. Ukr. J. Phys. 2020. Vol. 65, No. 12

\subsection{Numerical solution of the eigenproblem}

In this subsection, we discuss the details of the numerical solution of the eigenproblem. Eigenproblem (1) should be solved with proper boundary conditions at the lateral edges of the waveguide. We use a complete description of the dipolar interaction via Green's functions:

$\hat{\mathbf{G}}_{k_{x}}(y)=\frac{1}{2 \pi} \int_{-\infty}^{\infty} \hat{\mathbf{N}}_{k} e^{i k_{y} y} d k_{y}$

Here,

$\hat{\mathbf{N}}_{k}=\left(\begin{array}{ccc}\frac{k_{x}^{2}}{k^{2}} f(k h) & \frac{k_{x} k_{y}}{k^{2}} f(k h) & 0 \\ \frac{k_{x} k_{y}}{k^{2}} f(k h) & \frac{k_{y}^{2}}{k^{2}} f(k h) & 0 \\ 0 & 0 & 1-f(k h)\end{array}\right)$

where $f(k h)=1-(1-\exp (-k h)) / k h, k=\sqrt{k_{x}^{2}+k_{y}^{2}}$, and it is assumed that the waveguides are infinitely long.

The boundary condition (6) only accounts for the exchange interaction and surface anisotropy (if any) and reads [44]

$\mathbf{m} \times\left(\mu_{0} M_{\mathrm{s}} \lambda^{2} \frac{\partial \mathbf{m}}{\partial \mathbf{n}}-\nabla_{\mathbf{M}} E_{\mathrm{a}}\right)=0$

where $\mathbf{n}$ is the unit vector defining the inward normal direction to the waveguide edge, and $E_{\mathrm{a}}(\mathbf{m})$ is the energy density of the surface anisotropy. In the studied case of a waveguide magnetized along its long axis, conditions (6) for the dynamic magnetization components can be simplified to

$\pm \frac{\partial m_{y}}{\partial y}+\left.d m_{y}\right|_{y= \pm w / 2}=0,\left.\quad \frac{\partial m_{z}}{\partial y}\right|_{y= \pm w / 2}=0$

where $d=-2 K_{\mathrm{s}} /\left(m_{0} M_{\mathrm{s}}^{2} \lambda^{2}\right)$ is the pinning parameter [19], and $K_{\mathrm{s}}$ is the surface anisotropy constant at the waveguide lateral edges. More complex cases like, e.g., diffusive interfaces, can be considered in the same manner [45].

For the numerical solution of Eq. (1), it is convenient to use finite-element methods and to discretize the waveguide into $n$ elements of the width 
$\Delta w=w / n$, where $w$ is the width of the waveguide. The discretization step should be at least several times smaller than the waveguide thickness and the spin-wave wavelength $2 \pi / k_{x}$ for a proper description of the magneto-dipolar fields. The discretization transforms Eq. (1) into a system of linear equations for the magnetizations $\mathbf{m}_{j}, j=1,2,3, \ldots, n$

$$
\begin{aligned}
& i \boldsymbol{\mu} \times\left(\left(\omega_{\mathrm{M}}+\omega_{\mathrm{M}} \lambda^{2} k_{x}^{2}\right) \mathbf{m}_{j}-\right. \\
& -\omega_{\mathrm{M}} \lambda^{2} \frac{\mathbf{m}_{j-1}-2 \mathbf{m}_{j}+\mathbf{m}_{j+1}}{\Delta w^{2}}+ \\
& \left.+\omega_{\mathrm{M}} \sum_{j^{\prime}=1}^{n} \hat{\mathbf{G}}_{j-j^{\prime}} \cdot \mathbf{m}_{j^{\prime}}\right)=\omega \mathbf{m}_{j}
\end{aligned}
$$

where the dipolar interaction between the discretized elements is described by

$$
\begin{aligned}
& \hat{\mathbf{G}}_{k_{x}, j}(y)= \\
& =\frac{1}{\Delta w} \int_{-\Delta w / 2}^{\Delta w / 2} d y \int_{-\Delta w / 2}^{\Delta w / 2} d y^{\prime} \hat{\mathbf{G}}_{k_{x}}\left(y-y^{\prime}-j \Delta w\right) .
\end{aligned}
$$

The direct use of Eq. (9) is complicated, since Green's function $\hat{\mathbf{G}}_{k_{x}}(y)$ is an integral itself. Using the Fourier transformation, it can be derived as

$$
\hat{\mathbf{G}}_{k_{x}, j}(y)=\frac{\Delta w}{2 \pi} \int \operatorname{sinc}\left(k_{y} \Delta w / 2\right) \hat{\mathbf{N}}_{k} e^{i k_{y} j \Delta w} d k_{y},
$$

with $\operatorname{sinc}(x)=\frac{\sin (x)}{x}$. This can be easily calculated, especially using the fast Fourier transformation. Equation (8) is, in fact, a $2 n$-dimensional linear algebraic eigenproblem (since $\mathbf{m}_{j}$ is a 2-component vector), which is solved by standard methods. The values $\mathbf{m}_{0}$ and $\mathbf{m}_{n+1}$ in Eq. (8) are determined from the boundary conditions (7). In particular, for the negligible anisotropy at the waveguide edges, one should set $\mathbf{m}_{0}=\mathbf{m}_{1}$ and $\mathbf{m}_{n+1}=\mathbf{m}_{n}$.

\section{Results and Discussions}

\subsection{Original experimental findings}

In these studies, we consider rectangular magnetic waveguides as shown schematically in Fig. 1, $b$. In the experiment, a spin-wave mode is excited by a stripline that provides a homogeneous excitation field over the sample containing various waveguides etched from a $h=39 \mathrm{~nm}$ thick YIG film. The widths of the waveguides range from $w=50 \mathrm{~nm}$ to $w=1 \mu \mathrm{m}$ and the length is $60 \mu \mathrm{m}$. The waveguides are uniformly magnetized along their long axis by an external field $B$ ( $x$-direction). Figures $1, c$, and $1, d$ show scanning electron microscopy (SEM) micrographs of the largest and the narrowest waveguide studied in the experiment. The intensity of the magnetization precession is measured by microfocused BLS spectroscopy [46] (see the "Methodology" section) as shown in Fig. 1, $b$. Black and red lines in Fig. 3, $a$ show the frequency spectra for the $1 \mu \mathrm{m}$ and $50-\mathrm{nm}$ wide waveguides, respectively. No standing modes across the thickness were observed in our experiment, as their frequencies lie higher than $20 \mathrm{GHz}$ due to the small thickness. The quasi-FMR frequency is $5.007 \mathrm{GHz}$ for the $1 \mu \mathrm{m}$ wide waveguide. This frequency is comparable to $5.029 \mathrm{GHz}$, the value predicted by the classical theoretical model using the thin strip approximation [17-19]. In contrast, the quasi-FMR frequency is $5.35 \mathrm{GHz}$ for a $50 \mathrm{~nm}$ wide waveguide which is much smaller than the value of $7.687 \mathrm{GHz}$ predicted by the same model. The reason for this is that the thin strip approximation overestimates the effect of the dipolar pinning in waveguides with aspect ratio either $a_{\mathrm{r}}=1$ or close to one, for which the nonuniformity of the dynamic dipolar fields is not well-localized at the waveguide edges. Additionally, in such nanoscale waveguides, the dynamic magnetization components become of the same order of magnitude and both affect the effective mode pinning, in contrast to thin waveguides, in which the in-plane magnetization component is dominant.

\subsection{Spin pinning in nanostructures}

In the following, the experiment is compared to the theory and to micromagnetic simulations.

The bottom panels of Figs. 2, $a$ and $b$ show the spin-wave mode profile of the fundamental mode for $k_{x}=0$, which corresponds to the quasi-FMR, in the $1 \mu \mathrm{m}(a)$ and $50 \mathrm{~nm}(b)$ wide waveguides which have been obtained by micromagnetic simulations (blue dots) and by solving Eq. (1) numerically (black lines) (higher width modes are discussed in the next sections). The top panels illustrate the mode profile and the local precession amplitude in the waveguide. As it can be seen, the two waveguides feature quite different profiles of their fundamental modes: in the $1 \mu \mathrm{m}$ wide waveguide, the spins are partially pinned, and the amplitude at the edges of the waveg- 
uide is reduced compared to the maximal value of $m_{z}=1$. This still resembles the cosine-like profile of the lowest width mode $n=0$ that has been well established in investigations of the spin-wave dynamics in waveguides on the micron scale $[25,29,47]$ and that can be well-described by the simple introduction of a finite effective width $w_{\text {eff }}>w\left(w_{\text {eff }}=w\right.$ for the case of full pinning). In contrast, the spins at the edges of the narrow waveguide are completely unpinned, and the amplitude of the dynamic magnetization $m_{z}$ of the lowest mode $n=0$ is almost constant across the width of the waveguide, resulting in $w_{\text {eff }} \rightarrow \infty$.

To understand the nature of this depinning, it is instructive to consider the spin-wave energy as a function of the geometric width of the waveguide normalized by the effective width $w / w_{\text {eff }}$. This ratio corresponds to some kind of the pinning parameter taking values in between 1 for the fully pinned case and 0 for the fully unpinned case. According to Ritz's variational principle, the profiles of the spin wave modes correspond to the respective minima of the spin wave frequency (energy) functional. Since only one minimization parameter $-w_{\text {eff }}-$ is used, the minimization as a function of $w / w_{\text {eff }}$ yields only the approximate spin wave profiles. Nevertheless, this is sufficient for the qualitative understanding. To illustrate this, Figs. 2, $c$ and $d$ show the normalized square of the spin-wave eigenfrequencies $\omega^{\prime 2} / \omega_{M}^{2}$ for the two different widths as a function of $w / w_{\text {eff }}$. Here, $\omega^{\prime 2}$ refers to a frequency square, not accounting for the Zeeman contribution $\left(\omega_{\mathrm{H}}^{2}+\omega_{\mathrm{H}} \omega_{\mathrm{M}}\right)$, which only leads to an offset in frequency. The minimum of $\omega^{\prime 2}$ is equivalent to the solution with the lowest energy corresponding to the effective width $w_{\text {eff }}$. In addition to the total $\omega^{\prime 2}$ (black), the individual contributions from the dipolar term (red) and the exchange term (blue) are shown, which can only be separated conveniently from each other, if the square of Eq. (3) is considered for $k_{x}=0$. The dipolar contribution is nonmonotonic and features a minimum at a finite effective width $w_{\text {eff }}$, which can clearly be observed for $w=1 \mu \mathrm{m}$. The appearance of this minimum, which leads to the effect known as "effective dipolar pinning" $[18,19]$, is a result of the interplay of two tendencies: (i) an increase in the volume contribution with increasing $w / w_{\text {eff }}$, as for common Damon-Eshbach spin waves, and (ii) a decrease in the edge contribution, when the spin-wave amplitude at the edges vanishes $\left(w / w_{\text {eff }}>1\right)$. This minimum is also present in the case of the $50 \mathrm{~nm}$

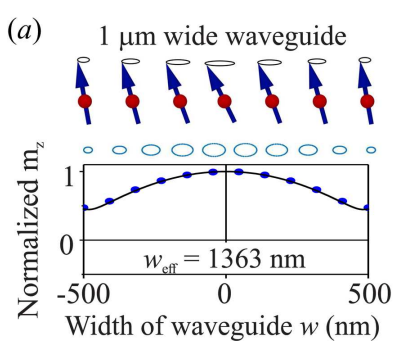

(c)

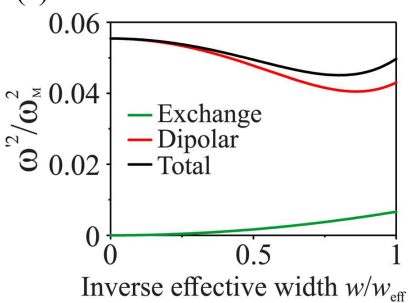

(b)
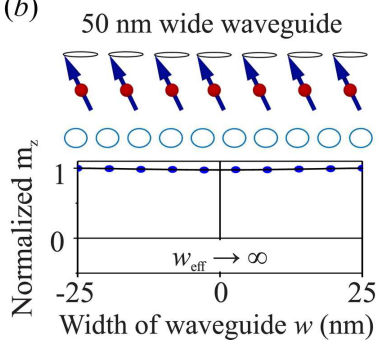

(d)

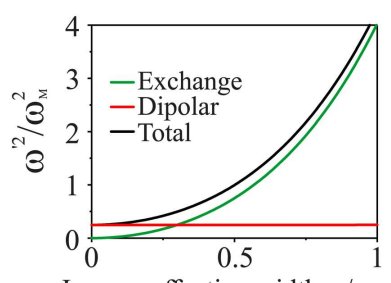

Inverse effective width $w / w_{\text {eff }}$

Fig. 2. Schematic of the precessing spins and simulated precession trajectories (ellipses in the second panel) and spin-wave profile $m_{z}(y)$ of the quasi-FMR. The profiles have been obtained by micromagnetic simulations (blue dots) and by the quasianalytic approach (black lines) for the $1 \mu \mathrm{m}(a)$ and $50 \mathrm{~nm}$ wide $(b)$ waveguides. Corresponding normalized square of the spin-wave eigenfrequency $\omega^{\prime 2} / \omega_{\mathrm{M}}^{2}$ as a function of $w / w_{\mathrm{eff}}$ and the relative dipolar and exchange contributions [31] $(c),(d)$

wide waveguide (red line), even though this is hardly perceivable in Fig. 2, $d$ due to the scale. In contrast, the exchange leads to a monotonic increase in the frequency as a function of $w / w_{\text {eff }}$, which is minimal for the unpinned case, i.e., $w / w_{\text {eff }}=0$ implying $w_{\text {eff }} \rightarrow \infty$, when all spins are parallel. In the case of the $50 \mathrm{~nm}$ waveguide, the smaller width and the corresponding much larger quantized wavenumber in the case of pinned spins would lead to a much larger exchange contribution than this is the case for the $1 \mu \mathrm{m}$ wide waveguide (please note the vertical scales). Consequently, the system avoids the pinning, and the solution with the lowest energy is situated at $w / w_{\text {eff }}=0$. In contrast, in the $1 \mu \mathrm{m}$ wide waveguide, the interplay of dipolar and exchange energies implies that the energy is minimized at a finite $w / w_{\text {eff }}$.

\subsection{Dependence of the spin-wave frequency on the spin pinning and the critical width of the exchange unpinning}

As is evident from Figs. 2, $c$ and 2, $d$, the pinning and the corresponding effective width have a large influence on the spin-wave frequency. This al- 
(a)

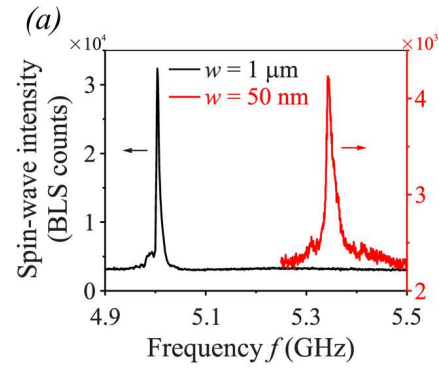

(b)

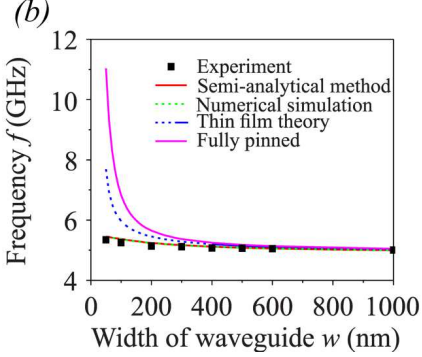

(c)

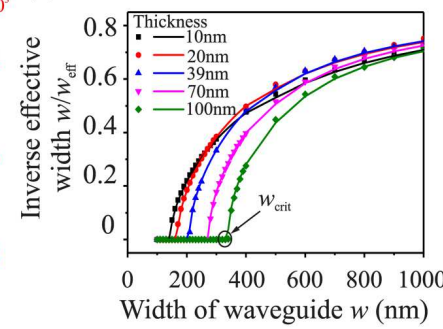

(d)

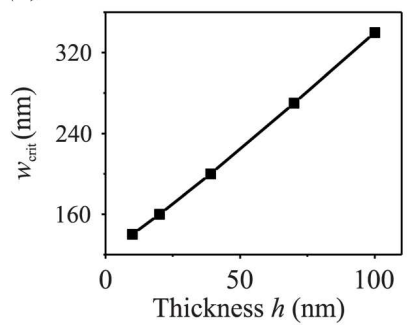

(e)

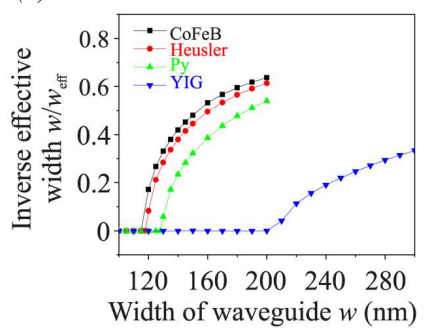

(f)

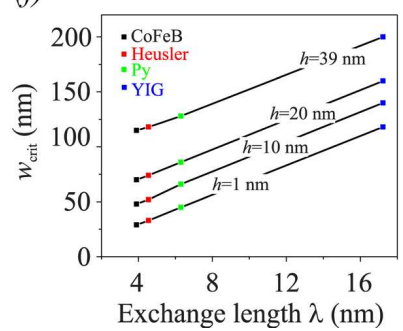

Fig. 3. Frequency spectra for the $1 \mu \mathrm{m}$ and $50 \mathrm{~nm}$ wide waveguides measured for respective microwave powers of $6 \mathrm{dBm}$ and $15 \mathrm{dBm}(a)$. Experimentally determined resonance frequencies (black squares) together with theoretical predictions and micromagnetic simulations $(b)$. Inverse effective width $w / w_{\text {eff }}$ as a function of the waveguide width $(c)$. The critical width $\left(w_{\text {crit }}\right)$ as a function of the thickness $h(d)$. Inverse effective width $w / w_{\text {eff }}$ as a function of the waveguide width for different materials at a fixed thickness of $39 \mathrm{~nm}(e)$. The critical width $w_{\text {crit }}$ as a function of the exchange length $\lambda$ for different thicknesses $(f)$. $(a)-(c)[31]$

lows for an experimental verification of the presented theory, since the frequency of partially pinned spinwave modes would be significantly higher than in the unpinned case. Black squares in Fig. 3, b summarize the dependence of the frequency of the quasiFMR on the width of the YIG waveguide. The magenta line shows the expected frequencies assuming pinned spins, the blue (dashed) line gives the resonance frequencies extrapolating the formula conventionally used for micron-sized waveguides [48] to the nanoscopic scenario, and the red line gives the result of the theory presented here, together with simulation results (green dashed line). As is seen, the experimentally observed frequencies can be well reproduced, if the real pinning conditions are taken into account.

As has been discussed alongside with Fig. 2, the unpinning occurs, when the exchange interaction contribution becomes so large that it compensates the minimum in the dipolar contribution of the spin-wave energy. Since the energy contributions and the demagnetization tensor change with the thickness of the investigated waveguide, the critical width below which the spins become unpinned is different for different waveguide thicknesses. This is shown in Fig. 3, c, where the inverse effective width $w / w_{\text {eff }}$ is shown for different waveguide thicknesses. Symbols are the results of micromagnetic simulations, lines are calculated semianalytically. As can be seen from the figure, the critical width linearly increases with the thickness. This is summarized in Fig. 3, $d$, which shows the critical width (i.e. the maximum width for which $\left.w / w_{\text {eff }}=0\right)$ as a function of the thickness.

The critical widths for YIG, Permalloy, CoFeB, and the Heusler compound $\mathrm{Co}_{2} \mathrm{Mn}_{0.6} \mathrm{Fe}_{0.4} \mathrm{Si}$ with different thicknesses are investigated. Figure 3,e shows the inverse effective width $w / w_{\text {eff }}$ as a function of the waveguide width for these materials which can be considered as typical materials used in magnonics. Figure $3, f$ shows the critical width $\left(w_{\text {crit }}\right)$ as a function of the exchange length $\lambda$ for different thicknesses. A simple empirical linear formula is found by fitting the critical widths for different materials in a wide range of thicknesses to estimate the critical width:

$w_{\text {crit }}=2.2 h+6.7 \lambda$

where $h$ is the thickness of the waveguide, and $\lambda$ is the exchange length given by $\lambda=\sqrt{2 A /\left(\mu_{0} M_{\mathrm{S}}^{2}\right)}$ with 
(a)

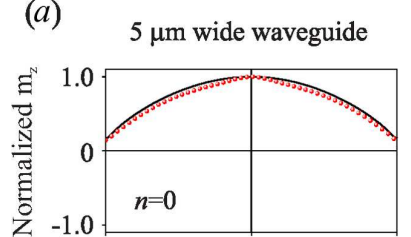

(b)
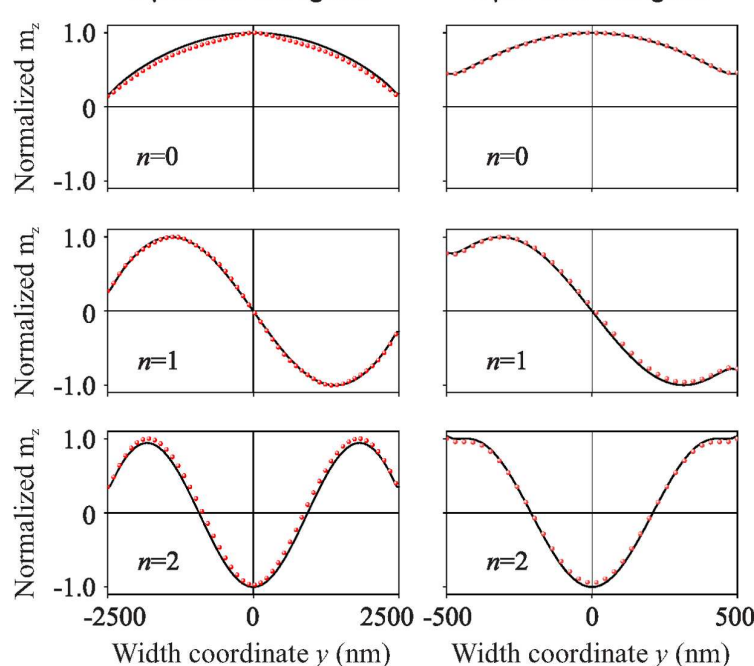

(c)

$50 \mathrm{~nm}$ wide waveguide
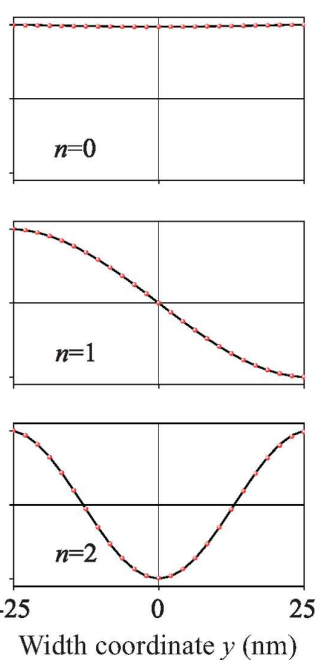

Fig. 4. Spin-wave profile representatively depicted using the $m_{z}$ component of the dynamic magnetization for the three lowest width modes obtained by the micromagnetic simulation (black solid lines) and numerical calculation (red dots) for (a) $5 \mu \mathrm{m}$,

(b) $1 \mu \mathrm{m}$ and (c) $50 \mathrm{~nm}$ wide waveguides, respectively

the exchange constant $A$, the vacuum permeability $\mu_{0}$, and the saturation magnetization $M_{\mathrm{s}}$.

\subsection{Profiles of higher-order width modes}

In [31], only the profile of the fundamental mode $(n=0)$ has been discussed. Therefore, the mode profiles of higher width modes are shown in Fig. 4 for the widths of the waveguides of $5 \mu \mathrm{m}$ corresponding to the practically fully pinned case (Fig. 4, a), $50 \mathrm{~nm}$ representing fully unpinned case (Fig. 4, c), and $1 \mu \mathrm{m}$ which can be considered as an intermediate case (Fig. $4, b$ ). For the $5 \mu \mathrm{m}$ wide waveguide, all higher width modes are clearly partially pinned due to the large width and an insufficient contribution of the exchange energy. In contrast to this, the higher modes of the $1 \mu \mathrm{m}$ wide waveguide are clearly unpinned for modes $n>2$. Since the fundamental mode is already unpinned for a $50 \mathrm{~nm}$ wide waveguide, also all higher width modes are fully unpinned.

\subsection{Temperature dependence of the spin pinning and frequencies of the spin-wave modes}

In the following, the quasianalytic theory is used to study the influence of the temperature on the discussed phenomena. There are two main parameters that introduce the temperature dependence of the spin-wave dispersion, the pinning condition and the pinning parameter: the saturation magnetization $M_{\mathrm{s}}$ and the exchange constant $A$. Furthermore, the temperature dependence of the surface anisotropy constant $K_{\mathrm{s}}$ at the lateral edges of the waveguide, can lead to an additional temperature dependence of the pinning parameter $d$ (see Eq. (7)). However, this dependence is typically rather weak and is, therefore, neglected in the following. The calculated saturation magnetization $M_{\mathrm{s}}$ for YIG is shown in Fig. 5, $a$ as a function of the temperature and was obtained using the theoretical model developed in [49]. The experimentally measured temperature dependence of the exchange constant $A$ taken from [50] is shown in Fig. 5, $b$.

Figure 5, $c$ shows the resulting temperature dependence of the frequencies of the first three modes for a YIG waveguide of the thickness $h=20 \mathrm{~nm}$, width $w=200 \mathrm{~nm}$, and for an external magnetic field $B_{0}=108.9 \mathrm{mT}$ applied along the stripe. One can clearly see that the frequencies of all modes decrease with the temperature due to a decrease in the saturation magnetization. The critical width, at which the unpinning takes place, depends on both the saturation magnetization and the exchange constant, as it can be seen, e.g., from the empirical Eq. (11). The 

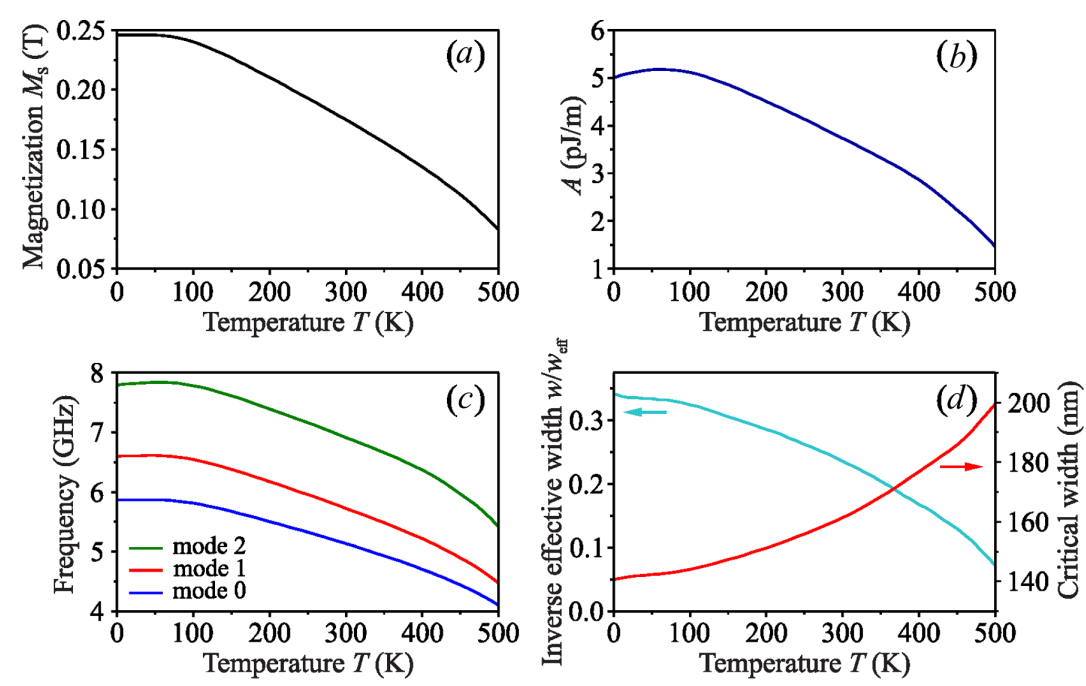

Fig. 5. Temperature dependence of the saturation magnetization $(a)$ and exchange constant (b) of YIG. (c) The temperature dependences of the frequencies of the three first modes for a YIG waveguide with $h=20 \mathrm{~nm}, w=200 \mathrm{~nm}$ and $B_{0}=108.9 \mathrm{mT}$. (d) The temperature dependence of the inverse effective width (left axis) and critical width of the exchange unpinning (right axis)

(a)

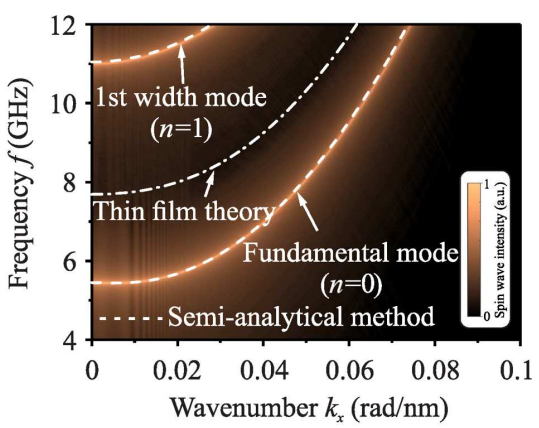

(b)

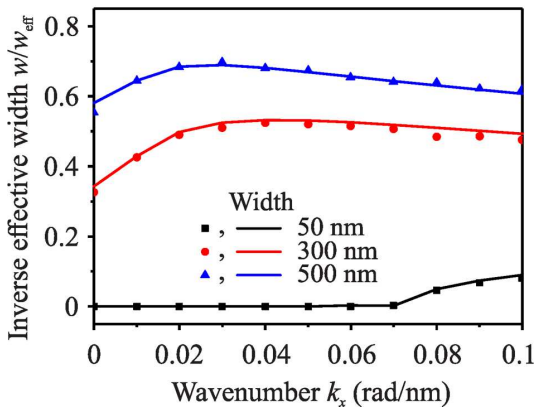

Fig. 6. (Spin-wave dispersion relation of two first width modes from micromagnetic simulations (color-code) and theory (dashed lines) (a). Inverse effective width $w / w_{\text {eff }}$ as a function of the spin-wave wavenumber $k_{x}$ for different thicknesses and waveguide widths, respectively (a) [31] interplay between both dependencies results in an increase in the critical width with temperature from a value of around $140 \mathrm{~nm}$ at zero temperature up to around $200 \mathrm{~nm}$ at $500 \mathrm{~K}$ - see Fig. 5, d. At the same time, the spin pinning, which is shown in the same figure in terms of the inverse effective width of the waveguide $w / w_{\text {eff }}$, decreases with the increase in temperature. This happens due to the dominant contribution of the temperature dependence of the saturation magnetization which, consequently, defines the strength of the dipolar pinning phenomenon. To conclude, if one conducts low temperature experiments which rely on or require a fully unpinned state of the system, a careful design of the structure dimensions is necessary.

\subsection{Spin-wave dispersion in nanostructures and the dependence of the spin pinning on the spin-wave wavenumber}

Up to now, the discussion was limited to the special case of $k_{x}=0$. In the following, the influence of the finite wave vector will be addressed. The spin-wave dispersion relation of the fundamental $(n=0)$ mode obtained from micromagnetic simulations (color-code) together with the semianalytic solution (white dashed line) are shown in Fig. 6, $a$ for the YIG waveguide 
of $w=50 \mathrm{~nm}$ in width. The figure also shows the low-wavenumber part of the dispersion of the first width mode $(n=1)$, which is pushed up significantly in frequency due to its large exchange contribution. Both modes are described accurately by the quasi-analytical theory. As it is described above, the spins are fully unpinned in this particular case. In order to demonstrate the influence of the pinning conditions on the spin-wave dispersion, a hypothetic dispersion relation for the case of partial pinning is shown in the figure with a dash-dotted white line (the case of $w / w_{\text {eff }}=0.63$ is considered that would result from the usage of the thin strip approximation [17]). One can clearly see that the spin-wave frequencies in this case are considerably higher. Figure $6, b$ shows the inverse effective width $w / w_{\text {eff }}$ as a function of the wavenumber $k_{x}$ for three exemplary waveguide widths of $w=50 \mathrm{~nm}, 300 \mathrm{~nm}$, and $500 \mathrm{~nm}$. As can be seen, the effective width and, consequently, the ratio $w / w_{\text {eff }}$ show only a weak nonmonotonic dependence on the spin-wave wavenumber in the propagation direction. This dependence is a result of an increase in the inhomogeneity of the dipolar fields near the edges for larger $k_{x}$, which increases the pinning [19], and of the simultaneous decrease of the overall strength of dynamic dipolar fields for shorter spin waves. Please, note that the mode profiles are not only important for the spin-wave dispersion. The unpinned mode profiles also greatly improve the coupling efficiency between two adjacent waveguides [9,51-53].

\subsection{Spin-wave lifetime in magnetic nanostructures}

The spin-wave lifetime depends on the ellipticity of the magnetization precession, and, thus, on the spin pinning conditions. The top panel in Fig. $2, b$ shows an additional feature of the narrow waveguide: as the aspect ratios of the waveguides approach unity, the ellipticity of the precession, a well-known feature of micron-sized waveguides which still resemble a thin film [29,44], vanishes and the precession becomes nearly circular. In addition, in nanoscale waveguides, the ellipticity is constant across the width, while it can be different at the waveguide center and near its edges for the $1 \mu \mathrm{m}$ wide waveguide. In general, the definition of the ellipticity $\epsilon$ of the precession is given by the ratio of the precession components as follows:

$\epsilon=1-\frac{m_{\min }}{m_{\max }}$,

ISSN 2071-0194. Ukr. J. Phys. 2020. Vol. 65, No. 12 where $m_{\min }$ and $m_{\max }$ denote the respective amplitudes of the smaller and larger components of the precession. Calculating the average relation between the magnetization components $m_{y}$ and $m_{z}$, we get

$\left|\frac{m_{y}}{m_{z}}\right|=\sqrt{\left(\frac{\left(\omega_{\mathrm{H}}+\omega_{\mathrm{M}}\left(\lambda^{2} K^{2}+F_{k_{x}}^{z z}\right)\right.}{\left(\omega_{\mathrm{H}}+\omega_{\mathrm{M}}\left(\lambda^{2} K^{2}+F_{k_{x}}^{y y}\right)\right.}\right)}$,

from which the ellipticity can be calculated for any width in dependence on the spin-wave wavenumber $k_{x}$, as is shown in Fig. 7, $a$.

The relaxation lifetime $\tau$ of the uniform precession mode in an infinite medium (without inhomogeneous linewidth $\left.\Delta B_{0}\right)$ is simply defined as $\tau=1 /(\alpha \omega)$, where $\omega$ is the angular frequency of the spin wave, and $\alpha$ is the damping. However, the dynamic demagnetizing field has to be taken into account in finite spin-wave waveguides. The lifetime can be found by the phenomenological model [54-56]

$\tau=\left(\alpha \omega \frac{\partial \omega}{\partial \omega_{\mathrm{H}}}\right)^{-1}$

The dispersion relation has been shown in Eq. (3). The demagnetization tensors are independent of $\omega_{\mathrm{H}}$. Differentiating Eq. (3) yields the lifetime as

$\tau=\left(\frac{1}{2} \alpha\left(2 \omega_{\mathrm{H}}+2 \omega_{\mathrm{M}} \lambda^{2} K^{2}+\omega_{\mathrm{M}}\left(F_{k_{x}}^{z z}+F_{k_{x}}^{y y}\right)\right)\right)^{-1}$

This formula clearly shows that the lifetime of the uniform precession $\left(k_{x}=0\right)$ depends only on the sum of the dynamic $y y$ and $z z$ components of the demagnetization tensors.

Figure $7, b$ shows the cross-section, spin precession trajectory (red line), and the dynamic components of the demagnetization tensors of different sample geometries. The spin precession trajectory changes from elliptic for a thin film $\left(a_{\mathrm{r}} \ll 1\right)$ to circular for a nanoscopic waveguide $\left(a_{\mathrm{r}}=1\right)$. The spin precession trajectory in the bulk material is also circular (in the geometry, where spin waves propagate parallel to the static magnetic field, the same geometry as studied for nanoscale waveguides). The dependence of the lifetime on the wavenumber is shown in Fig. 7, $c$ for YIG with the damping constant $\alpha=2 \times 10^{-4}$. The inhomogeneous linewidth is not

1103 


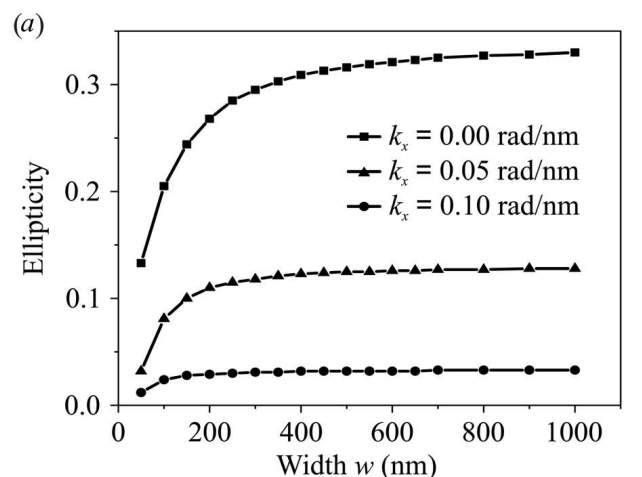

(b)
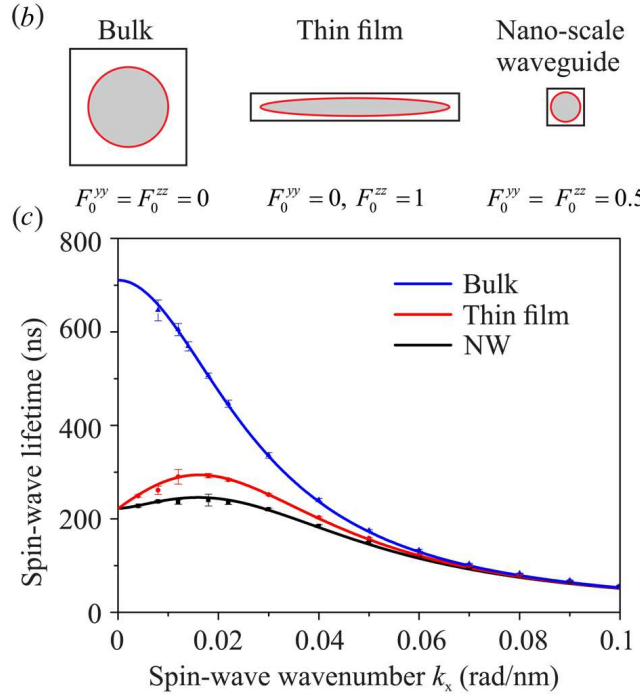

Fig. 7. Ellipticity as a function of the waveguide width for different spin-wave wavenumbers $k_{x}$ for the thickness $h=$ $=39 \mathrm{~nm}$ and the external magnetic field $B_{0}=108.9 \mathrm{mT}(a)$. The spin precession trajectories (red lines) and the components of the demagnetization tensor $F_{0}^{y y}$ and $F_{0}^{z z}$ for different sample geometries $(b)$. The spin-wave lifetime as a function of the spin-wave wavenumber. The lines and dots are obtained from Eq. (15) and the micromagnetic simulation, respectively (c)

taken into account. The lifetime of the uniform precession $\left(k_{x}=0\right)$ for the bulk material is much larger than that in a thin film and a nanoscopic waveguide, another consequence of the absence of the dynamic demagnetization in the bulk $\left(F_{0}^{z z}=F_{0}^{y y}\right)$. Moreover, the lifetimes of the uniform precession $\left(k_{x}=0\right)$ for a thin film (red line) and for a nanoscopic waveguide (black line) have the same value, because the lifetime depends only on the sum of the two components, which is the same for both cases.

Moreover, the $y y$ and $z z$ components of the demagnetization tensor decrease with an increase in the spin-wave wavenumber (instead, the $x x$ component, which does not affect the spin wave dynamic in our geometry, increases). The lifetime is inversely proportional to the square of the wavenumber and the sum of the dynamic demagnetization components. In the exchange region, the lifetime is, thus, dominated by the wavenumber. Therefore, the lifetimes for short-wave spin-waves are nearly the same for the three different geometries.

\subsection{Dependence of the spin pinning on a trapezoidal form of the waveguides}

A perfect rectangular form is not achievable in the experiment due to the involved patterning technique. As a result of the etching, the cross-section of the waveguides is always slightly trapezoidal. In this section, the influence of such a trapezoidal form on the spin pinning conditions is studied. In our experiment, the trapezoidal edges extent for approximately $20 \mathrm{~nm}$ on both sides for all the patterned waveguides, as can be seen from Fig. 1, d). We performed an additional simulation on waveguides with such trapezoidal edges. The simulated cross-section is shown in the top of Fig. 8. The thickness of the waveguide is divided into 5 layers with different widths ranging from $90 \mathrm{~nm}$ to $50 \mathrm{~nm}$. The steps at the edges are hard to be avoided due to the finite difference method used in MuMax3. The spin-wave profiles in the different $z$-layers are shown at the bottom of Fig. 8, $a$.

The results clearly show that the spin-wave profiles are fully unpinned along the entire thickness. This is due to the fact that the largest width $(90 \mathrm{~nm})$ is still far below the critical width. Hence, the influence of the trapezoidal form of the waveguide on the spin pinning condition is negligible for very narrow waveguides. For large waveguides, it also does not have a large impact as the ratio of the edge to the waveguide area becomes close to zero. Quantitatively, the quasiferromagnetic resonance frequency in the $50 \mathrm{~nm}$ wide waveguide decreases from $5.45 \mathrm{GHz}$ for the rectangular shape to $5.38 \mathrm{GHz}$ for the trapezoidal form due to the increase in the averaged width which is, in fact, even closer to the experimental results $(5.35 \mathrm{GHz})$.

The inverse effective width $w / w_{\text {eff }}$ as a function of the width of the waveguides is simulated for the trapezoidal and rectangular forms and the result is shown in Fig. 8, b. Here, the width is defined by the minimal width for the trapezoidal form, i.e., the width of the top layer. In the case of a trapezoidal

ISSN 2071-0194. Ukr. J. Phys. 2020. Vol. 65, No. 12 
(a)

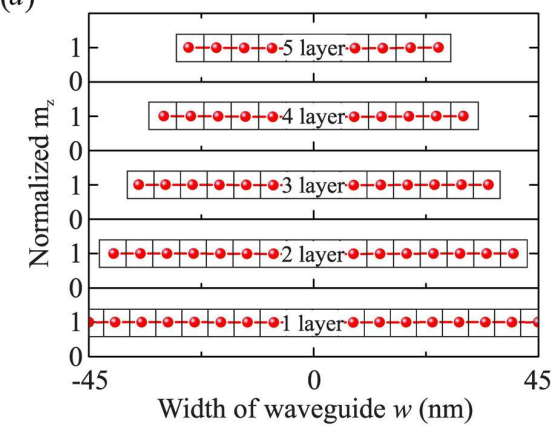

(b)

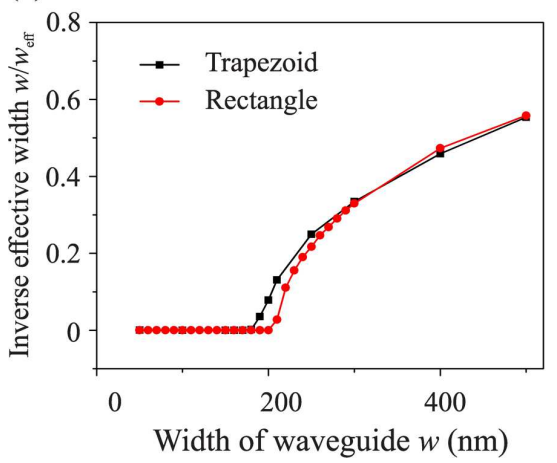

Fig. 8. Trapezoidal cross-section of the simulated waveguide with the normalized spin-wave profile for the different layers $(a)$. The inverse effective width $w / w_{\text {eff }}$ as a function of the width of the waveguide for trapezoidal and rectangular forms $(b)$

form, the inverse effective width is averaged over all 5 layers. The critical width slightly decreases from $200 \mathrm{~nm}$ for the rectangular cross-section to $180 \mathrm{~nm}$ for the trapezoidal form due to an increase in the averaged width. The difference between the inverse effective widths decreases with increasing width of the waveguide and vanishes, when the width is larger than $300 \mathrm{~nm}$.

Furthermore, it should be noted that the results of the multilayer simulations demonstrate that the assumption of a uniform dynamic magnetization distribution across the thickness that is used in our analytic theory and micromagnetic simulations featuring only one cell in the $z$ dimension is valid.

\subsection{Influence of edge roughness on the spin pinning}

Perfectly smooth edges are also hard to obtain in the experiment. Therefore, we have considered the influence of the edge roughness on the spin pinning. We

ISSN 2071-0194. Ukr. J. Phys. 2020. Vol. 65, No. 12 (a)

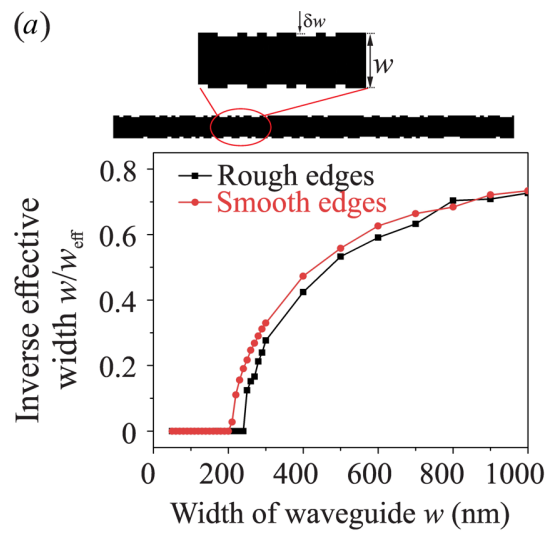

(b)

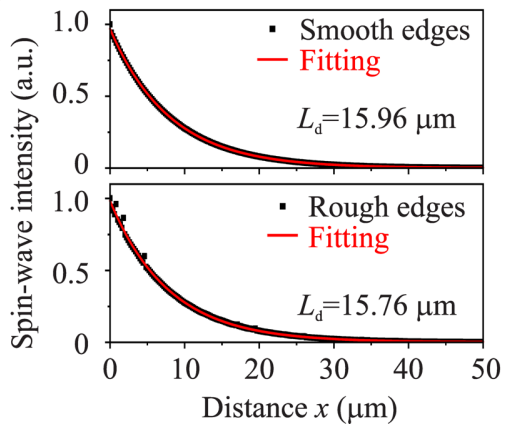

Fig. 9. Top: Schematic of the rough waveguide and close-up image of the introduced edge roughness. A single randomized defect pattern is generated for each structure width. Bottom: Inverse effective width $w / w_{\text {eff }}$ as a function of the waveguide width for rough and smooth edges $(a)$. The normalized spinwave intensity as a function of the propagation length for $50 \mathrm{~nm}$ wide waveguides with smooth and rough edges $(b)$

performed additional simulations on waveguides with rough boundaries for a fixed thickness of $39 \mathrm{~nm} .5 \mathrm{~nm}$ (for $50 \mathrm{~nm}$ to $100 \mathrm{~nm}$ wide waveguides) or $10 \mathrm{~nm}$ (for $100 \mathrm{~nm}$ to $1000 \mathrm{~nm}$ wide waveguides) wide rectangular nonmagnetic regions with a random length are introduced randomly on both sides of the waveguides to act as defects. The introduction of roughness results in a slight increase in the critical width from $200 \mathrm{~nm}$ to $240 \mathrm{~nm}$, as is shown in Fig. 9, a. These results demonstrate that the edge roughness does not have a large influence on the spin pinning condition.

Additional simulations are performed to study the influence of a rough edge on the propagation length of spin waves with a frequency of $6.16 \mathrm{GHz}\left(k_{x}=\right.$ $=0.03 \mathrm{rad} / \mathrm{nm})$. Figure $9, b$ shows the normalized spin-wave intensity as a function of the propagation length for smooth and rough-edged waveguides $50 \mathrm{~nm}$ 
in width. The decay length slightly decreases from $15.96 \mu \mathrm{m}$ for smooth edges to $15.76 \mu \mathrm{m}$ for rough edges. Since the spins in nanoscopic waveguides are already unpinned, the effect of such an edge roughness is not too important anymore, and the propagation length is essentially unaffected.

\section{Conclusions}

To conclude, an in-detail investigation of the pinning phenomenon based on the theoretical description of [31] is presented, and the quasianalytic model is outlined. The dependence of the effective width on the thickness and the material parameters is analyzed, and a simple empirical formula is found to predict the critical width for a given system. In addition to [31], higher order width modes up to $n=2$ are analyzed. An investigation of the effective width for finite wavevectors along the waveguide yields only a weak nonmonotonic dependence. It is shown that assuming a more realistic trapezoidal cross-section of the structures rather than the ideal rectangular shape results in a small decrease in the quasi-FMR frequency and a slight reduction in the critical width. Moreover, the influence of edge roughness is studied which shows a small increase in the critical width compared to the case of smooth edges. Here, the impact on the decay length of propagating waves is investigated as well, and only a small reduction is found. The temperature dependence of the pinning phenomenon shows that the dependences of the saturation magnetization and the exchange constant of YIG result in a decrease in the spin pinning with the increase in temperature and in an increase in the critical width of the exchange unpinning. This assumes that low temperatures are favorable for the dipolar pinning, and the sizes of the structures have to be decreased further in order to operate with fully unpinned uniform spin-wave modes.

The presented results provide valuable guidelines for applications in nano-magnonics, where spin waves propagate in nanoscopic waveguides with aspect ratios close to one and lateral sizes comparable to the sizes of modern CMOS technology.

The authors thank Burkard Hillebrands and Andrei Slavin for valuable discussions. This research has been supported by the European Research Council project ERC Starting Grant 678309 MagnonCircuits, by the Deutsche Forschungsgemeinschaft (DFG, Ger-

\section{6}

man Research Foundation) - 271741898, by the Collaborative Research Center SFB/TRR 173-268565370 (Project B01), and by the Austrian Science Fund (FWF) through the project I 4696-N. B.H. acknowledges support by the Graduate School Material Science in Mainz (MAINZ). R.V. acknowledges support by the National Academy of Sciences of Ukraine, grant No. 23-04/01-2019.

1. A.V. Chumak, V.I. Vasyuchka, A.A. Serga, B. Hillebrands. Magnon spintronics. Nat. Phys. 11, 453 (2015).

2. V.V. Kruglyak, S.O. Demokritov, D. Grundler. Magnonics. J. Phys. D 43, 264001 (2010).

3. C.S. Davies, A. Francis, A.V. Sadovnikov, S.V. Chertopalov, M.T. Bryan, S.V. Grishin, D.A. Allwood, Y.P. Sharaevskii, S.A. Nikitov, V. V. Kruglyak. Towards graded-index magnonics: Steering spin waves in magnonic networks. Phys. Rev. B 92, 020408 (2015).

4. A. Khitun, M. Bao, K.L. Wang. Magnonic logic circuits. J. Phys. D: Appl. Phys. 43, 264005 (2010).

5. M. Schneider, T. Brächer, D. Breitbach, V. Lauer, P. Pirro, D.A. Bozhko, H.Y. Musiienko-Shmarova, B. Heinz, Q. Wang, T. Meyer, F. Heussner, S. Keller, E.T. Papaioannou, B. Lägel, T. Löber, C. Dubs, A.N. Slavin, V.S. Tiberkevich, A.A. Serga, B. Hillebrands, A.V. Chumak. Bose-Einstein condensation of quasiparticles by rapid cooling. Nat. Nanotechnol. 15, 457 (2020).

6. M. Krawczyk, D. Grundler. Review and prospects of magnonic crystals and devices with reprogrammable band structure. J. Phys.: Condens. Matt. 26, 123202 (2014).

7. S. Wintz, V. Tiberkevich, M. Weigand, J. Raabe, J. Lindner, A. Erbe, A. Slavin, J. Fassbender. Magnetic vortex cores as tunable spin-wave emitters. Nat. Nanotechnol. 11, $948 \mathrm{EP}(2016)$.

8. T. Brächer, P. Pirro. An analog magnon adder for allmagnonic neurons. J. Appl. Phys. 124, 152119 (2018).

9. Q. Wang, P. Pirro, R. Verba, A. Slavin, B. Hillebrands, A.V. Chumak. Reconfigurable nanoscale spin-wave directional coupler. Sci. Adv. 4, e1701517 (2018).

10. O. Zografos, B. Soree, A. Vaysset, S. Cosemans, L. Amaru, P. Gaillardon, G. De Micheli, R. Lauwereins, S. Sayan, P. Raghavan, I.P. Radu, A. Thean. Design and benchmarking of hybrid cmos-spin wave device circuits compared to 10nm CMOS. In: Work-in-Progress session, 52nd Design Automation Conference (DAC), 7-11 June 2015, San Francisco, CA, USA (2015), pp. 686-689.

11. S. Manipatruni, D.E. Nikonov, I.A. Young. Beyond CMOS computing with spin and polarization. Nature Physics 14, 338 (2018).

12. A. Chumak. Fundamentals of magnon-based computing. arXiv 1901.08934 (2019).

13. Q. Wang, M. Kewenig, M. Schneider, R. Verba, B. Heinz, M. Geilen, M. Mohseni, B. Lägel, F. Ciubotaru, C. Adelmann, C. Dubs, S.D. Cotofana, T. Brächer, P. Pirro,

ISSN 2071-0194. Ukr. J. Phys. 2020. Vol. 65, No. 12 
A.V. Chumak. A magnonic directional coupler for integrated magnonic half-adders. Nat. Electron. (2020).

14. B.A. Ivanov, C.E. Zaspel. Magnon modes for thin circular vortex-state magnetic dots. Appl. Phys. Lett. 81, 1261 (2002).

15. G.T. Rado, J.R. Weertman. Spin-wave resonance in a ferromagnetic metal. J. Phys. Chem. Sol. 11, 315 (1959).

16. R.W. Damon, J.R. Eshbach. Magnetostatic modes of a ferromagnet slab. J. Phys. Chem. Sol. 19, 308 (1961).

17. K.Y. Guslienko, S.O. Demokritov, B. Hillebrands, A.N. Slavin. Effective dipolar boundary conditions for dynamic magnetization in thin magnetic stripes. Phys. Rev. $B$ 66, 132402 (2002).

18. K.Y. Guslienko, A.N. Slavin. Boundary conditions for magnetization in magnetic nanoelements. Phys. Rev. B 72, 014463 (2005).

19. K.Y. Guslienko, A.N. Slavin. Magnetostatic Green's functions for the description of spin waves in finite rectangular magnetic dots and stripes. J. Magn. Magnet. Mater. 323, 2418 (2011).

20. R.E. Arias. Spin-wave modes of ferromagnetic films. Phys. Rev. B 94, 134408 (2016).

21. B. Heinz, T. Brächer, M. Schneider, Q. Wang, B. Lägel, A.M. Friedel, D. Breitbach, S. Steinert, T. Meyer, M. Kewenig, C. Dubs, P. Pirro, A.V. Chumak, Propagation of spin-wave packets in individual nanosized yttrium iron garnet magnonic conduits. Nano Lett. 20, 4220 (2020).

22. T. Brächer, O. Boulle, G. Gaudin, P. Pirro. Creation of unidirectional spin-wave emitters by utilizing interfacial Dzyaloshinskii-Moriya interaction. Phys. Rev. B 95, 064429 (2017).

23. V.E. Demidov, S.O. Demokritov. Magnonic waveguides studied by microfocus Brillouin light scattering. IEEE Trans. Magnet. 51, 1 (2015).

24. F. Ciubotaru, T. Devolder, M. Manfrini, C. Adelmann, I.P. Radu. All electrical propagating spin wave spectroscopy with broadband wavevector capability. Appl. Phys. Lett. 109, 012403 (2016).

25. P. Pirro, T. Brächer, A. V. Chumak, B. Lägel, C. Dubs, O. Surzhenko, P. Gärnert, B. Leven, B. Hillebrands. Spinwave excitation and propagation in microstructured waveguides of yttrium iron garnet/Pt bilayers. Appl. Phys. Lett. 104, 012402 (2014).

26. M. Mruczkiewicz, P. Graczyk, P. Lupo, A. Adeyeye, G. Gubbiotti, M. Krawczyk. Spin-wave nonreciprocity and magnonic band structure in a thin permalloy film induced by dynamical coupling with an array of Ni stripes. Phys. Rev. B 96, 104411 (2017).

27. A. Haldar, A.O. Adeyeye. Deterministic control of magnetization dynamics in reconfigurable nanomagnetic networks for logic applications. ACS Nano 10, 1690 (2016).

28. R. Verba, V. Tiberkevich, E. Bankowski, T. Meitzler, G. Melkov, A. Slavin. Conditions for the spin wave nonreciprocity in an array of dipolarly coupled magnetic nanopillars. Appl. Phys. Lett. 103, 082407 (2013).
29. T. Brächer, P. Pirro, B. Hillebrands. Parallel pumping for magnon spintronics: Amplification and manipulation of magnon spin currents on the micron-scale. Phys. Rep. 699, 1 (2017).

30. G.S. Abo, Y. Hong, J. Park, J. Lee, W. Lee, B. Choi. Definition of magnetic exchange length. IEEE Trans. Magnet. 49, 4937 (2013)

31. Q. Wang, B. Heinz, R. Verba, M. Kewenig, P. Pirro, M. Schneider, M. Thomas, B. Lägel, C. Dubs, T. Brächer, A.V. Chumak. Spin pinning and spin-wave dispersion in nanoscopic ferromagnetic waveguides. Phys. Rev. Lett. 122, 247202 (2019).

32. Y. Tabuchi, S. Ishino, A. Noguchi, T. Ishikawa, R. Yamazaki, K. Usami, Y. Nakamura. Coherent coupling between a ferromagnetic magnon and a superconducting qubit. Science 349, 405 (2015).

33. O.V. Dobrovolskiy, R. Sachser, T. Brächer, T. Böttcher, V.V. Kruglyak, R.V. Vovk, V.A. Shklovskij, M. Huth, B. Hillebrands, A.V. Chumak. Magnon-fluxon interaction in a ferromagnet/superconductor heterostructure. Nat. Phys. 15, 477 (2019).

34. I. Golovchanskiy, N. Abramov, M. Pfirrmann, T. Piskor, J. Voss, D. Baranov, R. Hovhannisyan, V. Stolyarov, C. Dubs, A. Golubov, V. Ryazanov, A. Ustinov, M. Weides. Interplay of magnetization dynamics with a microwave waveguide at cryogenic temperatures. Phys. Rev. Appl. 11, 044076 (2019).

35. O.V. Prokopenko, D.A. Bozhko, V.S. Tyberkevych, A.V. Chumak, V.I. Vasyuchka, A.A. Serga, O. Dzyapko, R.V. Verba, A.V. Talalaevskij, D.V. Slobodianiuk, Y.V. Kobljanskyj, V.A. Moiseienko, S.V.Sholom, V.Y. Malyshev. Recent trends in microwave magnetism and superconductivity. Ukr. J. Phys. 64, 888 (2019).

36. L. Mihalceanu, D.A. Bozhko, V.I. Vasyuchka, A.A. Serga, B. Hillebrands, A. Pomyalov, V.S. L'vov, V.S. Tyberkevych. Magnon Bose-Einstein condensates and supercurrents over a wide temperature range. Ukr. J. Phys. 64, 927 (2019).

37. S. Kosen, A.F. van Loo, D.A. Bozhko, L. Mihalceanu, A.D. Karenowska. Microwave magnon damping in YIG films at millikelvin temperatures. APL. Mater. 7, 101120 (2019).

38. C. Dubs, O. Surzhenko, R. Thomas, J. Osten, T. Schneider, K. lenz, J. Grenzer, R. Hübner, E. Wendler. Low damping and microstructural perfection of sub-40nm-thin yttrium iron garnet films grown by liquid phase epitaxy. Phys. Rev. Materials 4, 024416 (2020).

39. I.S. Maksymov, M. Kostylev. Broadband stripline ferromagnetic resonance spectroscopy of ferromagnetic films, multilayers and nanostructures. Phys. E: Lowdimensional Systems and Nanostructures 69, 253 (2015).

40. A. Vansteenkiste, J. Leliaert, M. Dvornik, M. Helsen, F. Garcia-Sanchez, B. Van Waeyenberge. The design and verification of mumax3. AIP Advances 4, 107133 (2014). 
41. I.S. Maksymov, M. Kostylev. Broadband stripline ferromagnetic resonance spectroscopy of ferromagnetic films, multilayers and nanostructures. Physica E 69, 253 (2015).

42. R. Verba, G. Melkov, V. Tiberkevich, A. Slavin. Collective spin-wave excitations in a two-dimensional array of coupled magnetic nanodots. Phys. Rev. B 85, 014427 (2012).

43. R. Verba. Spin waves in arrays of magnetic nanodots with magnetodipolar coupling. Ukr. J. Phys. 58, 758 (2013).

44. A. Gurevich, G. Melkov. Magnetization Oscillations and Waves (CRC Press, 1996)

45. V.V. Kruglyak, O.Y. Gorobets, Y.I. Gorobets, A.N. Kuchko. Magnetization boundary conditions at a ferromagnetic interface of finite thickness. J. Phys.: Cond. Matt. 26, 406001 (2014).

46. T. Sebastian, K. Schultheiss, B. Obry, B. Hillebrands, H. Schultheiss. Micro-focused Brillouin light scattering: imaging spin waves at the nanoscale. Front. Phys. 3, 35 (2015).

47. M.B. Jungfleisch, W. Zhang, W. Jiang, H. Chang, J. Sklenar, S.M. Wu, J.E. Pearson, A. Bhattacharya, J.B. Ketterson, M. Wu, A. Hoffmann. Spin waves in micro-structured yttrium iron garnet nanometer-thick films. J. Appl. Phys. 117, 17D128 (2015).

48. B.A. Kalinikos, A.N. Slavin. Theory of dipoleexchange spin wave spectrum for ferromagnetic films with mixed exchange boundary conditions. J. Phys. C 19, 7013 (1986).

49. P. Hansen, P. Röschmann, W. Tolksdorf. Saturation magnetization of gallium-substituted yttrium iron garnet. J. Appl. Phys. 45, 2728 (1974).

50. R.C. LeCraw, L.R. Walker. Temperature dependence of the spin-wave spectrum of yttrium iron garnet. J. Appl. Phys. 32, S167 (1961).

51. A.V. Sadovnikov, E.N. Beginin, S.E. Sheshukova, D.V. Romanenko, Y.P. Sharaevskii, S.A. Nikitov. Directional multimode coupler for planar magnonics: Sidecoupled magnetic stripes. Appl. Phys. Lett. 107, 202405 (2015).

52. A.V. Sadovnikov, A.A. Grachev, S.E. Sheshukova, Y.P. Sharaevskii, A.A. Serdobintsev, D.M. Mitin, S.A. Nikitov. Magnon straintronics: Reconfigurable spinwave routing in strain-controlled bilateral magnetic stripes. Phys. Rev. Lett. 120, 257203 (2018).

53. A.V. Sadovnikov, S.A. Odintsov, E.N. Beginin, S.E. Sheshukova, Y.P. Sharaevskii, S.A. Nikitov. Toward nonlinear magnonics: Intensity-dependent spin-wave switching in insulating side-coupled magnetic stripes. Phys. Rev. B 96, 144428 (2017).

54. D.D. Stancil. Phenomenological propagation loss theory for magnetostatic waves in thin ferrite films. J. Appl. Phys. 59, 218 (1986).

55. D.D. Stancil, A. Prabhakar. Spin Waves. Theory and Applications (Springer, 2009).

56. R. Verba, V. Tiberkevich, A. Slavin. Damping of linear spin-wave modes in magnetic nanostructures: Local, nonlocal, and coordinate-dependent damping. Phys. Rev. B 98, 104408 (2018).

Received 15.07.20

Б. Хайни, К. Вонг, Р. Верба, В.І. Васючка,

М. Кевеніг, Ф. Пірро, М. Шнайдер, Т. Мейер, Б. Лагел,

К. Дабс, Т. Брачер, О.В. Доброволъсъкий, А.В. Чумак

ТЕМПЕРАТУРНА ЗАЛЕЖНІСТЬ

ЗАКРІПЛЕННЯ СПІНІВ ТА ДИСПЕРСІЇ

СПІНОВИХ ХВИЛЬ У НАНОРОЗМІРНИХ

ФЕРОМАГНІТНИХ ХВИЛЕВОДАХ

$\mathrm{P}$ е $з$ ю м е

На сьогодні область магноніки привертає значну увагу завдяки можливості кодування інформації за допомогою фази чи амплітуди спінової хвилі та подальшій обробці на нанометровому масштабі. Нещодавно були досліджені спінові хвилі в хвилеводах залізо-ітрієвого гранату (ЗІГ) із шириною до 50 нм і відношенням товщини до ширини, що наближається до одиниці. Знайдено критичну ширину, нижче якої обмінна взаємодія пригнічує явище дипольного закріплення спінів і профілі мод стають вільними. У даній статті ми продовжуємо ці дослідження та аналізуємо дисперсію спінових хвиль і явище закріплення спінів як функції температури, матеріальних параметрів та товщини хвилеводу. Обговорюються особливості вищих мод, вплив скінченного хвильового числа вдовж хвилеводу, трапецієвидного перерізу хвилеводу та шорсткості країв на дисперсію спінових хвиль, а також час життя спін-хвильових мод. Представлені результати становлять особливий інтерес для актуальної області квантової магноніки при кріогенних температурах. 